\title{
The Effect of Network's Size on the Performance of the Gateway Discovery and Selection Scheme for MANEMO
}

\author{
Zainab S. Mahmod, Aisha Hassan A. Hashim, Othman O. Khalifa, Farhat Anwar, Shihab \\ A. Hameed \\ Faculty of Engineering, International Islamic University Malaysia, $53100 \mathrm{KL}$, Malaysia \\ e-mail: aisha@iium.edu.my
}

\begin{abstract}
In the era of Internet technology, new applications are developed everyday requiring continuous and seamless connections. This urges for access availability solutions to the new scenarios. One of the critical architecture is the Mobile Ad-Hoc Network Mobility (MANEMO). However, the integration of Ad-hoc and NEMO technologies came out with many complications like redundant tunnels and the existence of multiple Exit Routers. This paper presents a scheme to discover and select the optimum gateway to improve the robustness and the performance of the network irrespective of the used routing protocol. The MANEMO Gateway discovery and selection scheme (MGDSS) extends the Tree Discovery Protocol and the Neighborhood Discovery protocol used by NEMO and Ad-Hoc to carry the necessary gateway selection parameters. To compare the effect of network's size on the performance of the proposed scheme, the standard NEMO BSP and the Multi-homed MANEMO (M-MANEMO) approaches OPNET Modeler 14.5 was used. The results show that the average data packets dropped, the end-to-end delay and the throughput of the proposed MGDSS outperform those for the standard M-MANEMO and standard NEMO BSP.
\end{abstract}

Keywords: Gateway Selection, Mobile Ad Hoc NEMO, MANEMO, Network Mobility, MANET

\section{Introduction}

The Internet users and applications around the world are increasing in number. This is increasing the need of Internet resources and access points for different topologies and scenarios. To improve the network access in public transport (i.e. trains, buses, planes, and ships) the IETF presented Network Mobility Basic Support Protocol (NEMO BSP) [1]. Whereas, Mobile Ad-hoc Network (MANET) [2] was efficient for different topologies like Personal Area Networks (PANs) and Vehicle Ad hoc Networks (VANETs). Each as its advantages and drawbacks, therefore, [3] presented an integration between NEMO technology and the (MANETs) known as (MANEMO) to form scalable, global reachable, optimized network topologies.

Different researches were done to study and solve MANEMO's challenges. When a direct connection exists between the nodes this reduces latency. The MANET gives the node a chance to use the ad-hoc connection instead of communicating throughout the Mobile Routers (MRs) as the packets should go through many Home agents (HAs). However, changing to a direct connection is not always efficient. Therefore, efficient switching decision should be used to select the path in MANEMO.

[4] presented an efficient multi-path selection approach for MANEMO (eMANEMO) to use it in Vehicle-to-Vehicle (V2V) network. The path quality is used for selecting the best route between NEMO and MANET to achieve the highest performance for V2V communication network.

[5] presented the Unified MANEMO Architecture (UMA) which is a protocol architecture designed to support the different MANEMO models. The Unified MANEMO Architecture (UMA) gives a solution using two protocols for Nested NEMO scenarios: Network-In-NodeAdvertisement (NINA) protocol and the Tree Discovery (TD) protocol. The TDP broadcasts information among the MRs to allow them form optimal, loop-less tree topologies. While NINA sends route information through the tree topologies formed by the TD process. The two protocols TDP and NINA were developed as extensions of Neighbor Discovery (ND) process.

The used Network Address Translation (NAT) is used by [6] as a key element to avoid redundant paths in NAT-MANEMO. NAT is used to guarantee the global reachability of each 
MR in the MANET. While the MRs use the addresses advertised by the IGW as its own CoA using the routing protocols. However, NAT has many drawbacks as the address translation is limited to the address of the MR, leaving packets from the end node (MNN, Mobile Network Node) untouched.

MRs are required to select the Internet gateway in a way that maximizes the performance. There has been no standard mechanism for intelligent gateway selection in MANEMO. The multiple exits' problem can be solved if additional information related to the exit routes is carried to the MRs along with the route information. The Multi-homed MANEMO (MMANEMO) proposed by [7] merges two protocols: MCoA and MANEMO to support different multi-homed and mobility operations. M-MANEMO enables a multi-homed mobile tree and multiple gateways crossing the tree to provide heterogeneous Internet access. It also enables a gateway to have a NEMO home registration and tunneling to join the tree over additional egress interface.

This paper discusses the effect of network size on the performance of the MANEMO Gateway Discovery and Selection Scheme (MGDSS) [8] compared to M-MANEMO and NEMO BSP. The paper is organized as follows: section 2 states the design of the proposed MGDSS. Section 3 shows the performance evaluation of the proposed scheme in different network's sizes compared with NEMO BS, and Multi-homed MANEMO. That is followed by a discussion about the obtained results. Finally section 4 concludes the research findings.

\section{The Design of MGDSS}

The proposed MANEMO Gateway discovery and selection scheme MGDSS modified the NDP [9] to broadcast the performance information of the gateways to the mobile network nodes (MNNs) and the MRs. Those information will be propagated periodically to a certain number of hopes, the nodes beyond that number should send gateway request message. The gateways performance metrics will be stored at the nodes' gateway cache to be used when needed The MNNs connected with the NEMO structure will have the NEMO MRs information as well. The stored information at the MNN about the MANET gateways and the NEMO MRs are shown in Table 1 and 2 respectively.

Table 1. Information kept by MNs cache about MANET GWs

\begin{tabular}{ll}
\hline Information Field & Description \\
\hline GW global address & $\begin{array}{l}\text { The unique address for the gateway, can be used to send packets } \\
\text { to the Internet }\end{array}$ \\
GW MANET address & $\begin{array}{l}\text { The address used by the GW for MANET's internal } \\
\text { communication }\end{array}$ \\
The next hop & \\
The ling information & $\begin{array}{l}\text { The MANET will consider that the gateway information changed } \\
\text { after this time and should refresh them }\end{array}$ \\
GW's evaluation & $\begin{array}{l}\text { The criteria to evaluate the GW's capacity, stability, distance and } \\
\text { delivery delay }\end{array}$ \\
parameters & Number of hops between the node and the GW \\
Hop count &
\end{tabular}

Table 2. Information kept by MNs cache about NEMO MRs

\begin{tabular}{ll}
\hline \multicolumn{1}{c}{ Information Field } & Description \\
\hline MR's global Address & The MR's unique address \\
MR's Care of Address (CoA) & The address registered at its home agent \\
Routing information & Next hop \\
MR's evaluation parameters & The criteria to evaluate the GW's capacity, stability, \\
& distance and delivery delay \\
Hop count & The number of hops between the node and the MR \\
MR's nested level & The level in the tree topology \\
\hline
\end{tabular}

The Corresponding Node, the HAs and the MANET's routing protocols are not to be modified in this approach which make it easy to deploy. The modified TDP (M-TCP) and 
modified NHDP (M-NHDP) [10] are using tree information option of the IPv6 Neighbor Discovery Router Advertisement messages to broadcast the gateway information.

Switching from NEMO tree to MANET path can reduce network latency, and provide a high throughput route for the mobile nodes. However, if the mobility of MANET nodes is high, or the MANET is large in size, the unnecessary switching will cause overhead to the entire network. Even when the connectivity with the gateway is on but with high cost, the mobile node would switch to another optimized gateway to improve the performance. Furthermore, the MR may lose the connectivity to the NEMO tree, and select the MANET gateway as a default gateway. Therefore, the switching decision should be made after measuring and calculating the cost of all possible options to get the optimum path to the corresponding node. Different criteria for selecting the gateway are discussed in [11]. The chosen criteria for MGDSS are: hope count, NEMO nested-level, number of registered nodes, and Route Stable Time.

Using the above mentioned parameters, the selection decision is made using the Simple Additive Weighting (SAW) [12] method's calculation. This method uses multiple criteria and gives each of them its importance weight. The selected gateway is the one with minimum weight:

$$
\mathrm{GW}_{\text {selected }}=\operatorname{Min}\left\{\mathrm{GW}_{\mathrm{j}}\right\}
$$

And to calculate $\mathrm{GWj}$ the following equation is used:

$$
\mathrm{GW}_{\mathrm{j}}=(\mathrm{HC} \times \mathrm{n} \%)+(\mathrm{NN} \times \mathrm{m} \%)+(\mathrm{ST} \times \mathrm{k} \%)+(\mathrm{NL} \times \mathrm{p} \%)
$$

Where,

$\mathrm{GW}_{\mathrm{j}}$ is the weighted values of the gateway

$\mathrm{HC}$ is the hop count between the mobile node and the Gateway

$\mathrm{NN}$ is the number of nodes registered at the gateway

ST is the stable time of the route to the gateway

$\mathrm{NL}$ is the nested level of the MR, and equal to 1 for the MANET gateway

$\mathrm{n}, \mathrm{m}, \mathrm{k}$ and $\mathrm{p}$ are the respective weights given to $\mathrm{HC}, \mathrm{NN}, \mathrm{ST}$ and $\mathrm{NL}$

$n+m+k+p=100 \%$

These parameters' weights can be adjusted by the network administrator to give any of the parameters more importance based on the network preferences.

\section{Performance Evaluation}

The proposed scheme was evaluated into different network's size to test its scalability beside the robustness. The chosen scenarios were varied in the number of MANET Nodes and the NEMO Nesting-level. The simulation of the network using MGDSS, M-MANEMO and NEMO BSP is deployed with low traffic load and heavy traffic load. Figure 1 shows that WLAN throughput of the M-MANEMO scheme is $28.6 \%$ better compared to NEMO BSP in a scalable network. This is because of the large number of nodes between the MN and the $\mathrm{CN}$. The NEMO tree is a better route when the MANET is large in scale. The MGDSS outperforms the NEMO BSP with $69.2 \%$ since the proposed scheme is using a MANET path beside the NEMO tree. 


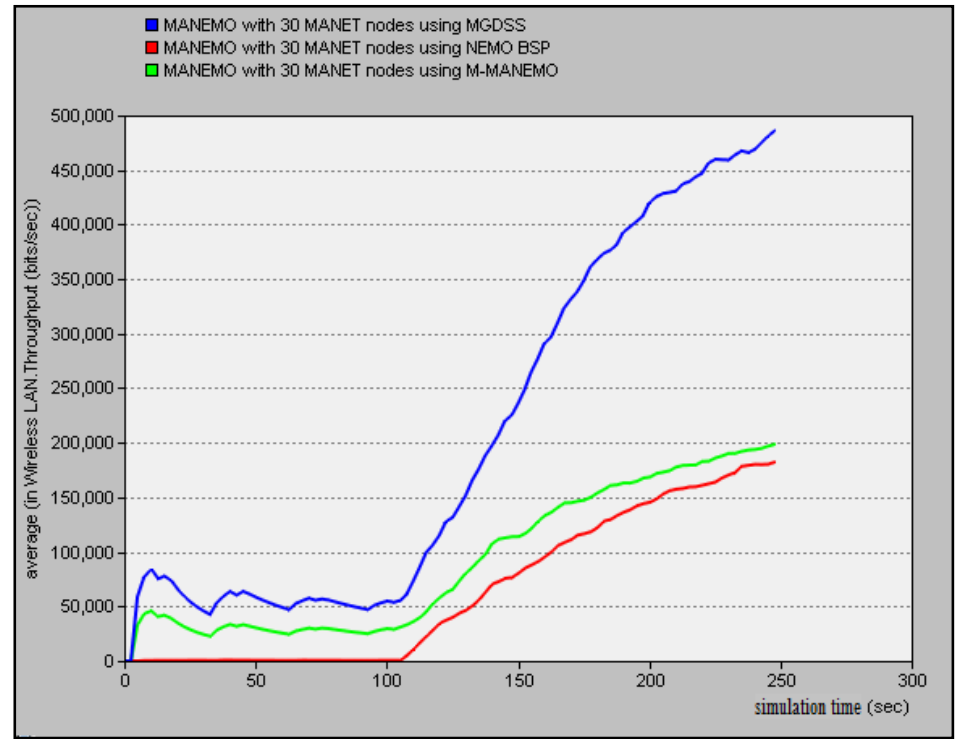

Figure 1. Average WLAN throughput in MANEMO with 30 MANET nodes

As the number of nesting level increases, the MGDSS performs better compared to MMANEMO and NEMO BSP. Figure 2 shows the average data packets dropped for MGDSS, MMANEMO and NEMO BSP. The data packets dropped of MGDSS improved $46.5 \%$ compared to M-MANEMO. This is due to the less number of MR-HA tunnels used in MGDSS.

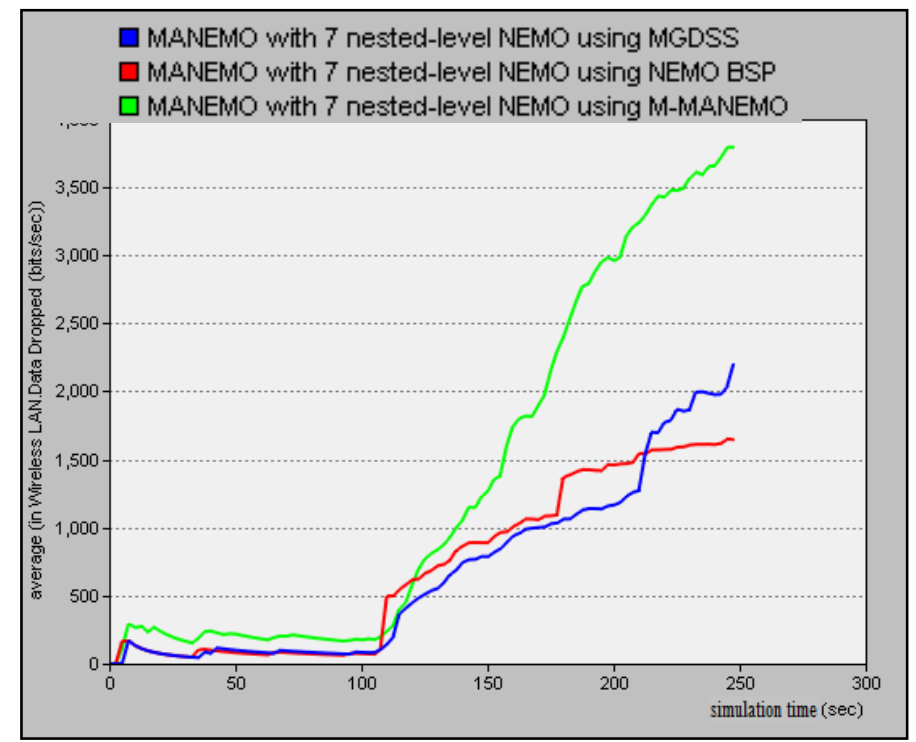

Figure 2. Average WLAN data packets dropped in MANEMO with 7 nested-level NEMO

In terms of Ethernet delay of MANEMO, the MGDSS outperforms M-MANEMO and NEMO BSP with improvement equals $9 \%$ and $10.8 \%$ respectively. Figure 3 shows the average Ethernet delay in MANEMO with 7 nested-level NEMO. 


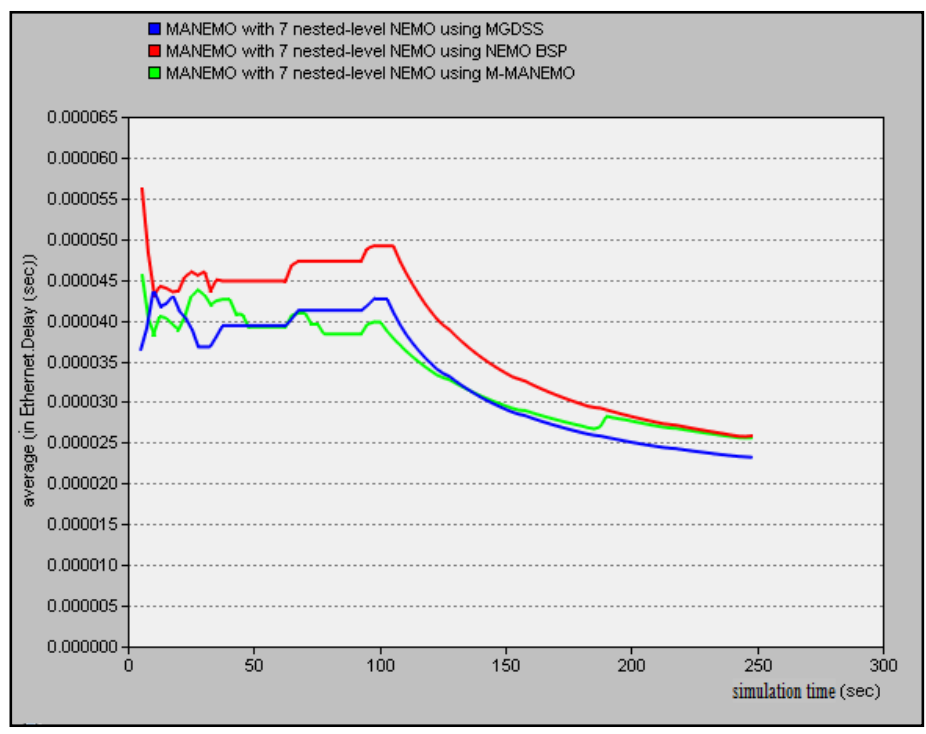

Figure 3. Average Ethernet delay in MANEMO with 7 nested-level NEMO

From the simulation results analysis, it is clear that there are different behaviors of the MANEMO and its gateways depending on the size of the network. In a small MANEMO scenario, the average data packets dropped in WLAN connecting the MRs of the proposed scheme shows $28.6 \%$ improvement compared to the NEMO BSP. This is because of the less number of hops and tunnels the packets are going through. It is also due to the re-routing process in the MANET when the intermediate nodes move out of the transmission range of their neighbors. The light weight given to the mobility selection parameter in the M-MANEMO causes higher delay.

In this section, it is explained the results of research and at the same time is given the comprehensive discussion. Results can be presented in figures, graphs, tables and others that make the reader

\section{Conclusion}

In this research, the performance of the gateway discovery and selection scheme for MANEMO is evaluated. The selection criteria used which are: Hop count, Nesting level, number of registered neighbors, and the stable time are having significant effect on the performance. The evaluation of the proposed scheme is done using OPNET simulator. The simulation performance parameters show that the MANEMO could be more scalable as the performance of the network is better than those fo M-MANEMO and NEMO BSP in large scale network.

\section{References}

[1] Ernst T. Network Mobility Support Goals and Requirements. IETF RFC4886, https://tools.ietf.org/html/rfc4886. 2007.

[2] Clausen T., Dearlove C., \& Dean J. Mobile Ad Hoc Network (MANET) Neighborhood Discovery Protocol (NHDP). IETF RFC6130, https://tools.ietf.org/html//fc6130. 2011.

[3] McCarthy B., Edwards C., \& Dunmore M. Advances in MANEMO: Definition of the Problem Domain and the Design of a NEMO-Centric Approach, Proceedings of the 2007 International Symposium on Applications and the Internet Workshops.

[4] Van Do T., Uehara K., Sato M., and Murai J. eMANEMO: An Efficient Multi-path Selection Method for MANEMO Applying to Vehicle-to-Vehicle Communication Network. IPSJ SIG Technical Report. 2011.

[5] McCarthy B., Edwards C., \& Dunmore M. Using NEMO to Extend the Functionality of MANETs, IEEE International Conference on Communications Workshops, 2008. ICC Workshops '08. IEEE. $2008 ; 455-460$. 
[6] Tazaki H, Van Meter R, Wakikawa R, Uehara K, Murai J. NAT-MANEMO: Route optimization for unlimited network extensibility in MANEMO. Journal of Information Processing. 2011; 19: 118-128.

[7] Alsukayti I. S., Edwards C. Efficient Mobility and Multihoming Support for Mountain Rescue, IFIP Wireless and Mobile Networking Conference (WMNC). 2015: 128 - 135

[8] Zainab S. Mahmod, AishaHassan A. Hashim, Othman O. Khalifa, Farhat Anwar, Shihab A. Hameed. Development of Gateway Discovery and Selection Scheme for MANEMO (MGDSS) . International Journal of Future Generation Communication and Networking (IJFGCN). 2017.

[9] Gashinsky I., Jaeggli J., \& Kumari W. Operational Neighbor Discovery Problems. IETF RFC6583, https://tools.ietf.org/html/rfc6583. 2012.

[10] Dearlove C, Clausen T. An Optimization for the Mobile Ad Hoc Network (MANET) Neighborhood Discovery Protocol (NHDP). IETF RFC7466, https://tools.ietf.org/html/rfc7466. 2015.

[11] Mahmood Z, Abdalla AH, Anwar F. Parameters Used in Gateway Selection Schemes for Internet Connected MANETs: A Review, World Academy of Science, Engineering and Technology, International Science Index 97. International Journal of Computer, Electrical, Automation, Control and Information Engineering; 9(1): 360 - 364.

[12] Zhao J, Yang X, Liu H. Load-balancing Strategy of Multi-gateway for Ad hoc Internet Connectivity. Proceedings of the International Conference on Information Technology: Coding and Computing (ITCC'05). 2005; 2: 592- 596. 